\title{
Spectral Triples in Particle Physics
}

\author{
Arkadiusz Bochniak ${ }^{1, \star}$ \\ ${ }^{1}$ Institute of Physics, Jagiellonian University, prof. Stanisława Łojasiewicza 11, 30-348 Kraków, Poland
}

\begin{abstract}
We give an overview of the approach to the Standard Model of Particle Physics and its extensions based on the Noncommutative Geometry. The notion of spectral triples is introduced and their applications in particle physics are presented. We revisit known results based on different approaches within Noncommutative Geometry, list problems which appeared in these methods, propose possible solutions and indicate future directions of research.
\end{abstract}

\section{Introduction}

The classical field-theoretical description of the Standard Model of Particle Physics is rather complicated and it is not so easy to see at first sight hidden fundamental structures behind such formalism. On the other hand we know that there are physical theories like General Relativity or classical mechanics which are described in a geometrical language, using the notion of Riemannian or symplectic manifolds, respectively. There is a natural question if there exists some purely geometrical description of the Standard Model. It turns out that it is possible but we have to extend the notion of geometry. To do that we use the idea of Noncommutative Geometry (NCG) which is based on the observation made by Gelfand and Naimark, known as the Gelfand-Naimark duality theorem, that the category of locally compact Hausdorff spaces is anti-equivalent to the category of commutative $C^{*}$-algebras. It means that the information about the topology of our space can be encoded in a properly choosen algebra. This algebra, for the case of a compact space $M$, is just the $C^{*}$-algebra $C(M)$ of continuous $\mathbb{C}$-valued functions on $M$. The idea of using functions on space instead of the space is nothing strange for physicists, because in physics we really manipulate functions defined on a space instead of using its points. This dual picture of geometry indicates its natural extension, mainly we can consider noncommutative $C^{*}$-algebras and think of them as a set of functions on noncommutative topological space, thus we get the notion of noncommutative topology. Due to the Gelfand-Naimark-Segal construction any $C^{*}$-algebra $\mathcal{A}$ is isometrically isomorphic to a $C^{*}$-algebra $B(H)$ of bounded operators on some Hilbert space $H$, i.e. there exists a faithful representation $\pi: \mathcal{A} \rightarrow B(H)$. Let us also mention one important fact, mainly the Serre-Swan theorem about the equivalence of the category of complex vector bundles on a compact Hausdorff space $X$ and the category of finite projective modules over the algebra $C(X)$. The vast majority of physical objects are just sections of some bundles (e.g. vector fields, spinors, etc.) or are objects defined in terms of bundles (connections, strength tensors, etc.), therefore projective modules over noncommutative $C^{*}$-algebras are natural candidates for noncommutative vector bundles.

\footnotetext{
^e-mail: arkadiusz.bochniak@student.uj.edu.pl
} 
Now, let us consider the differential part of the theory. The knowledge of the topology is not enough to have the whole set of information about the geometry. Consider a Dirac operator on closed, oriented Riemannian manifold that admits spinors, i.e. the operator given in local coordinates by $\not D_{M}=i \gamma^{\mu}\left(\partial_{\mu}+\omega_{\mu}\right)$, where $\gamma^{\mu}$ are $\gamma$-matrices and $\omega_{\mu}$ can be expressed by Levi-Civita connection lifted to act on spinor-valued functions. The Dirac operator determines the metric structure [4] and is the crucial ingredient in the construction of K-homology classes. The Dirac operator $D_{M}$ together with $C^{\infty}(M)$ and a Hilbert space $L^{2}(M, S)$ of square-integrable spinors form a canonical example of a spectral triple - an object which we will define precisely in the next section, where we will demonstrate how to apply such a concept in case of SM and explain how to search for its possible extensions.

\section{Spectral triples and the Standard Model}

\subsection{Spectral triples}

From now on, the geometry will be understood as a unital spectral triple $(\mathcal{A}, H, \mathcal{D})$ defined as in [7], i.e. $\mathcal{A}$ is an unital involutive algebra (not necessary a $C^{*}$-algebra) representable as bounded operators on a separable Hilbert space $H$, and $\mathcal{D}$ is a selfadjoint operator on $H$ with compact resolvent such that $a \cdot \operatorname{Dom}(\mathcal{D}) \subset \operatorname{Dom}(\mathcal{D})$ and $[\mathcal{D}, a]$ extends to a bounded operator on $H$, for all $a \in \mathcal{A}$. Moreover, we will always assume that our spectral triple is even and real, i.e. there exists grading operator $\gamma \in \operatorname{End}(H), \gamma^{*}=\gamma, \gamma^{2}=1$ that commutes with all elements of the algebra and anticommutes with the Dirac operator, and there exists $J \in \operatorname{Isom}(H)$ that is antilinear and satisfies

$$
J^{2}=\varepsilon 1, J \mathcal{D}=\varepsilon \mathcal{D} J, J \gamma=\varepsilon^{\prime \prime} \gamma J
$$

with $\varepsilon, \varepsilon^{\prime}, \varepsilon^{\prime \prime} \in\{ \pm 1\}$ dependent on the KO-dimension [8]. Moreover, there are two important conditions which have to be satisfied for all $a$ and $b$ :

- $0^{\text {th }}$ order condition : $\left[a, J b J^{-1}\right]=0$,

- $1^{\text {st }}$ order condition : $\left[[\mathcal{D}, a], J J^{-1}\right]=0$.

The canonical spectral triple $\left(C^{\infty}(M), L^{2}(M, S), \not D_{M}\right)$ which was previously mentioned is a natural example of this general concept. Another class are finite spectral triples, which can be classified [15] [18].

\subsection{The Standard Model}

Our goal is to use the notion of spectral triples to explore the structure of the Standard Model of Particle Physics. To do that observe that the gauge group $U(1) \times S U(2) \times S U(3)$ describes (up to extra $U(1)$ term) the unitary elements of the following finite-dimensional algebra

$$
\mathcal{A}_{s m}=\mathbb{C} \oplus \mathbb{H} \oplus M_{3}(\mathbb{C}),
$$

where $\mathbb{H}$ is the algebra of quaternions. Therefore $\mathcal{A}_{s m}$ is a natural choice for the first ingredient of a spectral triple. The choice of a Hilbert space is dictated by the number of particles we have in the Standard Model. Simple counting [10] implies the dimensionality equal 96. Taking into account chiralities of particles and existance of antiparticles the Hilbert space can presented as a direct sum

$$
H_{s m}=H_{L} \oplus H_{R} \oplus H_{L}^{c} \oplus H_{R}^{c}
$$

Therefore, the Dirac operator must be a $96 \times 96$-matrix that has to satisfy required conditions. Moreover, we also define the grading operator $\gamma$ to be just the usual chirality operator and the real 
structure $J$ which acts interchanging particle and antiparticle sectors and taking complex conjugation. The existence of mentioned compatibility conditions implies that the Dirac operator has a lot of zeros in its entries. The nonzero values are related to the Yukawa couplings, Majorana masses of neutrinos, CKM and PMNS mixing matrices [10].

To get some numbers from this construction, which can be experimentally tested, we consider almost-commutative spectral triples with an algebra $C^{\infty}(M) \otimes \mathcal{A}_{s m}$, where $M$ plays a role of a background for our theory of particles. The Hilbert space is the tensor product of Hilbert spaces we had on each part. Similarly, as tensor products, we construct grading $\Gamma$ and the real structure on the product spectral triple. Note that the form of the last one follows from the values of KO-dimensions [8]. The Dirac operator is defined by

$$
\mathcal{D}=\not D_{M} \otimes 1+\gamma_{5} \otimes \mathcal{D}_{s m},
$$

where $D_{M}$ is the usual Dirac operator on spin manifold $M, \mathcal{D}_{s m}$ is the Dirac operator we had in the spectral triple for the Standard Model and $\gamma_{5}$ is the usual grading on $M$.

For a given spectral triple we have the space of 1 -forms

$$
\Omega_{\mathcal{D}}^{1}(\mathcal{A})=\left\{\sum_{k} a_{k}\left[\mathcal{D}, b_{k}\right], a_{k}, b_{k} \in \mathcal{A}\right\}
$$

and we can use them to construct fluctuations of the Dirac operator by taking

$$
\mathcal{D}_{A}=\mathcal{D}+A+J A J^{-1}
$$

To obtain some physical quantities we use the spectral action

$$
S=\frac{1}{2}\left\langle J \xi, \mathcal{D}_{A} \xi\right\rangle+\operatorname{Tr} f\left(\frac{\mathcal{D}_{A}}{\Lambda}\right),
$$

where $\xi \in \frac{1}{2}\left(1+\gamma_{5} \otimes \gamma\right) H, f$ is a smooth approximation of the characteristic function of the interval $[0,1]$ and $\Lambda$ is a cutt-off parameter. Using heat kernel expansion [2] we can calculate the second part in powers of $\Lambda^{-1}$. After collecting terms in first order we get the bosonic part of action of the Standard Model on the curved background given by a manifold $M$, i.e. with Hilbert-Einstein terms but also with a coupling of Higgs field to the Ricci scalar. This approach gives us an effective Lagrangian at some energy scale $\Lambda$, on which all gauge coupling constants are equal. Assuming so-called Big Desert Hypothesis and using the standard tools of renormalization group we can explore the behaviour of running coupling constants and $\beta$-functions. This allows us to get values of these parameters at lower scales. Moreover, in the input we had only parameters of fermions and the bosonic fields appear from the inner fluctuations, therefore we can express the bosonic parameters in terms of fermionic one, for example we can calculate the mass of Higgs boson and the value we obtained is $\sim 170 \mathrm{GeV}$ [2] which is near the experimental one $(\sim 126 \mathrm{GeV})$, but different. Possible explanations of the source of this difference and another problems in the Standard Model will be discussed in the next section. Let us emphasize here that the NCG reveals the origin of the spontaneous symmetry breaking in the Standard Model.

\subsection{Problems}

There are few problems related to the previously mentioned results. First of all, the difference in the Higgs mass suggest that we are on the right way to explain the structure of the Standard Model on the fundamental, purely geometrical level, but still something has to be improved. Moreover, 
the low value of the Higgs mass is related to another problem of the instability of the electroweak vacuum known as Higgs vacuum problem. Another important issue is the question if the assumption of the Big Desert is adequate. From the most actual data we know that the extrapolated values of gauge couplings do not meet at exactly one point, but they rather form a triangle, so the predicted property of SM following from NCG is only approximately satisfied. Additional problems are related to the presence of right-handed neutrinos in the model, which Majorana mass terms can cure some of the previous problems, but we encounter the issue of see-saw mechanism, the same problem as in the classical approach to the SM. Another problems arise from considerations of Majorana-type particles. There are also some other questions related to the choice of specific mathematical structures what we will briefly discuss in the next section.

\section{Extensions of the Standard Model}

Problems mentioned in the previous section suggest not only that something has to be improved, but also indirectly indicate the way how to do it [9]. The problem of the difference in Higgs mass can be cured by adding additional fermions to the theory. The vacuum instability can be repaired by the introduction of a new $\sigma$-field, which was done many years ago in the particle physics in the context of Weinberg-Salam theory. One possibility to incorporate such a field in NCG approach is to change by hand the Yukawa coupling related to right handed neutrino Majorana mass $y_{R}$ into a field $y_{R} \sigma(x)$. As a result we cure the instability and additionally get the proper value of Higgs mass, but in NCG scalar fields should be produced from fluctuations of Dirac operator, so we have to take another spectral triple or relax some of the axioms to get the $\sigma$-field in purely geometric way. If we fix all ingredients of the spectral triple unless an algebra, then the more general form of the last constituent is [10],[9]

$$
M_{a}(\mathbb{H}) \oplus M_{2 a}(\mathbb{C}), \text { where } a \in \mathbb{N} .
$$

The existence of the grading implies that $a \geq 2$. For the smallest possible case, i.e. $a=2$, one can proof [10] that requiring commutation relation between the grading and the algebra the last one is reduced to

$$
\mathbb{H}_{L} \oplus \mathbb{H}_{R} \oplus M_{4}(\mathbb{C}) .
$$

If, in addition, we require the $1^{\text {st }}$-order condition then we get the algebra $\mathcal{A}_{s m}$ of the Standard Model. Therefore, the Standard Model is just the smallest possible noncommutative manifold. One can consider the exact form of the representation of this algebra on Hilbert space $H_{s m}$ and try to find possible generalizations or extension of the SM. If we want to save number of fermions in our theory and do not relax any condition of spectral triple, then the only possibility, due to dimensionality argument, is to introduce mixing of spinorial and gauge indices and take larger algebra $M_{2}(\mathbb{H}) \oplus M_{4}(\mathbb{C})$ - this choice is called Grand Symmetry model [10]. Repeating all steps done in case of the SM one can get $\sigma$-field which follows from fluctuations of Dirac operator in a similar way as the Higgs field [11]. Moreover, in that approach we can go back into the Standard Model by imposing additional condition, but this last symmetry breaking is not given by a dynamical mechanism [9]. Nevertheless, the idea of mixing spinorial and gauge indices is important one for study possible extensions of SM. The problem of symmetry breaking from Grand Symmetry into the SM can be solved by using twisted version of Dirac operator as was proposed in [12]. Nowadays, we have more general notion of twisted spectral triples, which can be use as an alternative approach to study particle physic models by the consideration of so-called Twisted Standard Model [16]. Moreover, from the general considerations we know that in the NCG approach there is no room for $S U(5)$ or $S O(10)$ GUTs and we have some kind of no-go theorem for supersymmetry at least in the case of MSSM [1]. We can also ask if our axioms of noncommutative manifolds are adequate. We can, for example, relax $1^{\text {st }}$-order condition, 
then for the smallest noncommutative manifolds we end up with a family of Pati-Salam models with $S U(2)_{L} \times S U(2)_{R} \times S U(4)$ gauge group. In such models the unification scale is of the order $10^{16} \mathrm{GeV}$ and the symmetry breaking to the SM appears at $m_{R}=10^{11}-10^{13} \mathrm{GeV}$, depending on the details of the model [3]. It is also a scale for the see-saw mechanism. Symmetries of Dirac operator in such case provide us with the existence of few Higgs-like fields and in the simplest, so-called composite, Pati-Salam model we get additional field in Higgs sector which lowers the value of Higgs mass to the value consistent with experiment. We can also consider other possible changes of algebraic conditions for spectral triples, especially introduction of $2^{\text {nd }}$-order condition related to Clifford structure and the aspects of Morita equivalence for spectral triples [7],[6] or consider more deeply other concepts like leptoquark models[17] and the role of the $S^{0}$-symmetry [5],[15]. Moreover, we can also take into account possible changes in the structure of almost-commutative geometries by replacing the standard tensor product by the graded one [13]. Another approaches based on Loretzian spectral triples are also considered [14] and provide alternative framework to study such phenomena.

\section{Summary}

We presented a short overview of alternative approach to the description of particle physics based on the notion of spectral triples and discussed main results, issues and indications for possible extensions and future searches. We should use our knowledge from experiments to perform some kind of reverse engineering to find proper geometric description of particle physics, especially by analysing the algebraic structure of finite spectral triples and their modifications.

\section{References}

[1] W. Beenakker, T. van den Broek, W. D. van Suijlekom, Supersymmetry and Noncommutative Geometry (Springer, Cham, 2016) 136

[2] A. H. Chamseddine, A. Connes, M. Marcolli, Adv. Theor. Math. Phys. 11, 991-1089 (2007)

[3] A. H. Chamseddine, A. Connes, W. D. van Suijlekom, J. High Energ. Phys. 11 (2015)

[4] A. Connes, Ergod. Theory Dyn. Syst. 9, 207-220 (1989)

[5] A. Connes, J. Math. Phys. 36, 6194 (1995)

[6] F. D’Andrea, L. Dạbrowski, J. Noncommut. Geom. 10, 551-578 (2016)

[7] F. D'Andrea, L. Dạbrowski, A. Sitarz, arXiv:1703.05279v1 [math-ph]

[8] L. Dąbrowski, G. Dossena, Int. J. Geom. Methods Mod. Phys. 8, 1833-1848 (2010)

[9] A. Devastato, J. Phys.: Conf. Ser. 634012008 (2015)

[10] A. Devastato, F. Lizzi, P. Martinetti, J. High Energy Phys. 01042 (2014)

[11] A. Devastato, F. Lizzi, P. Martinetti, Fortsch.Phys. 62 863-868 (2014)

[12] A. Devastato, P. Martinetti, Math. Phys. Anal. Geom. 20 (2017)

[13] S. Farnsworth, J. Math. Phys. 58, 023507 (2017)

[14] N. Franco, arXiv:1710.10959 [math-ph]

[15] T. Krajewski, J. Geom. Phys. 28, 1-30 (1998)

[16] G. Landi, P. Martinetti, Lett. Math. Phys. 106 no.11, 1499-1530 (2016)

[17] M. Paschke, F. Scheck, A. Sitarz, Phys. Rev. D 59, 035003 (1999)

[18] M. Paschke, A. Sitarz, J. Math. Phys. 396191 (1998) 